\title{
Electronic Spectra of Polyacetylenes
}

\author{
Michael BeER* \\ Department of Physics, University of Michigan, Ann Arbor, Michigan $\dagger$
}

(Received November 4, 1955)

\begin{abstract}
The low-temperature emission spectra in rigid glass solutions of several substituted polyacetyelnes have been investigated. All the compounds phosphoresced with decay lifetimes ranging from 0.01 to 0.3 second. None of the compounds with less than four triple bonds fluoresced except diphenyl acetylene. All compounds with four triple bonds did fluoresce. An explanation is offered for this phenomenon. Some new absorption measurements were made on dimethyl diacetylene, dimethyl triacetylene, and dimethyl tetraacetylene. The polarization of the transition between 32000 and $40000 \mathrm{~cm}^{-1}$ in a single crystal of dimethyl triacetylene was found to be along the axis of the molecules. These results and those of previous investigators show that the energy level arrays of the molecules can be related and that they vary systematically as the number of triple bonds increases. This is in accord with expectation on the basis of simple molecular orbital theory.

An assignment of the symmetries of the electronic wave functions is proposed on the basis of the positions and intensities of the bands.
\end{abstract}

\section{INTRODUCTION}

$\mathrm{O}^{\mathrm{N}}$ $\mathrm{N}$ account of their simplicity, the polyacetylenes $R-(\mathrm{C} \equiv \mathrm{C})_{\mathrm{N}}-R^{\prime}$ are among the most important compounds in the study of molecular structure and molecular spectra. Their conjugated systems of triple bonds are linear in the ground state and involve only carbon atoms. ${ }^{1}$

Until recently only the first two homologs had been synthesized and studied. However, through the researches of Jones and co-workers, ${ }^{2}$ polyacetylenes with as many as six triple bonds in conjugation have become accessible, and a systematic examination of their spectra became possible.

In such an undertaking there are two stages. First, the excited electronic energy levels of the members of the homologous series must be determined. Then these must be "explained"; that is a correspondence must be established between the experimental results and the predictions made by an approximate theory. In this paper some new observations are reported and an attempt is made to interpret the experimental results.

\section{PREVIOUS WORK}

The results of previous investigations are summarized in Table I. For many of the bands no intensity determinations are available, but the workers have stated the pressures at which the spectra were studied. As a rough approximation we shall assume that

$$
p l f=C,
$$

where $p$ is the pressure at which the bands were studied, $l$ is the path length, and $f$ is the oscillator strength of the transition. $C$ is a constant; the same for all transitions.

Such a formula cannot be very accurate. First of all,

\footnotetext{
* Present address: National Research Council of Canada, Ottawa, Canada.

$\dagger$ The majority of the experimental work reported here was done in the Department of Chemistry, University of Manchester, Manchester, England.

1 G. A. Jeffrey and J. S. Rollett, Nature 166, 475 (1950).

${ }^{2}$ Cook, Jones, and Whiting, J. Chem. Soc. (London) 1952, 2005, $2010,2014,2883$.
}

it is not always stated whether appearance pressures or higher pressures are given. Secondly, the widths of the bands may be so different that the appearance pressure is a bad measure of the intensity. Clearly for the narrow bands the prediction would be relatively low. Nevertheless, in the absence of more detailed information, this

TABLE I. Previous work on electronic spectra of polyacetylenes.

\begin{tabular}{|c|c|c|c|c|c|}
\hline Compound & Band & $\mathrm{cm}^{-1}$ & Phase & Intensity & Ref. \\
\hline Acetylene & $\begin{array}{l}1 \\
2 \\
3 \\
4 \\
5\end{array}$ & $\begin{array}{l}42280 \\
49650 \\
54820 \\
65790 \\
74510\end{array}$ & $\begin{array}{l}\text { Vapor } \\
\text { Vapor } \\
\text { Vapor } \\
\text { Vapor } \\
\text { Vapor }\end{array}$ & $\begin{array}{l}10^{-4} \\
5 \times 10^{-4} \mathrm{a} \\
5 \times 10^{-2} \mathrm{a} \\
6 \times 10^{-2} \mathrm{~b} \\
17 \times 10^{-2} \mathrm{o}\end{array}$ & $\begin{array}{l}\text { d } \\
\text { e } \\
f \\
g, h \\
g, h\end{array}$ \\
\hline Diacetylene & $\begin{array}{l}6 \\
7 \\
8 \\
9\end{array}$ & $\begin{array}{l}34960 \\
40160 \\
60790 \\
69126\end{array}$ & $\begin{array}{l}\text { Vapor } \\
\text { Vapor } \\
\text { Vapor } \\
\text { Vapor }\end{array}$ & $\begin{array}{l}3 \times 10^{-5} \text { : } \\
7 \times 10^{-4} \\
\text { Very strong } \\
\text { Very strong }\end{array}$ & $\begin{array}{l}\mathrm{i} \\
\mathrm{i} \\
\mathbf{j} \\
\mathbf{j}\end{array}$ \\
\hline \multirow[t]{2}{*}{$\begin{array}{l}\text { Dimethyl } \\
\text { diacetylene }\end{array}$} & $7 a$ & 40000 & \multirow{2}{*}{$\begin{array}{l}\text { Ethanol } \\
\text { solution } \\
\text { Vapor }\end{array}$} & & $\mathrm{k}$ \\
\hline & $\begin{array}{r}10 \\
8\end{array}$ & $\begin{array}{l}52600 \\
59984\end{array}$ & & Strong & $\underset{\mathbf{j}}{\mathbf{j}}$ \\
\hline \multirow[t]{2}{*}{$\begin{array}{l}\text { Dimethyl } \\
\text { triacetylene }\end{array}$} & 11 & 32700 & \multirow{2}{*}{$\begin{array}{l}\text { Ethanol } \\
\text { solution } \\
\text { Ethanol } \\
\text { solution }\end{array}$} & \multirow[t]{2}{*}{$5 \times 10^{-3}$} & $\mathrm{k}$ \\
\hline & 12 & 48300 & & & $\mathrm{k}$ \\
\hline \multirow[t]{2}{*}{$\begin{array}{l}\text { Dimethyl } \\
\text { tetraacetylene }\end{array}$} & 13 & 28200 & \multirow{2}{*}{$\begin{array}{l}\text { Ethanol } \\
\text { solution } \\
\text { Ethanol } \\
\text { solution }\end{array}$} & $7 \times 10^{-3}$ & $\mathbf{k}$ \\
\hline & 14 & 42700 & & 3 & $\mathbf{k}$ \\
\hline \multirow[t]{2}{*}{$\begin{array}{l}\text { Dimethyl } \\
\text { pentaacetylene }\end{array}$} & 15 & 25400 & Ethanol & $10^{-2}$ & $\mathrm{k}$ \\
\hline & 16 & 38400 & $\begin{array}{l}\text { Ethanol } \\
\text { solution }\end{array}$ & 3.4 & $\mathrm{k}$ \\
\hline $\begin{array}{l}\text { Dimethyl } \\
\text { hexaacetylene }\end{array}$ & 17 & 35200 & $\begin{array}{l}\text { Ethanol } \\
\text { solution }\end{array}$ & 4.45 & k \\
\hline
\end{tabular}

a $f$ estimated from stated working pressure using relation (1).

b First perpendicular Rydberg band.

- First parallel Rydberg band.

d See reference 12 .

- See reference 9

See reference 8.

b G. Moe and A. B. F. Duncan, J. Am. Chem. Soc. 74, 3136 (1952).

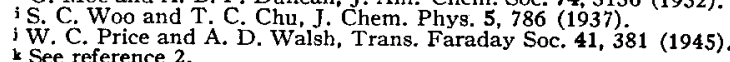


TABLE II. The fluorescence bands of some polyacetylenes.

\begin{tabular}{|c|c|c|c|}
\hline $\begin{array}{c}\text { Diethyl } \\
\text { tetraacetylene } \\
\mathrm{cm}^{-1}\end{array}$ & $\begin{array}{c}\text { Tetraacetylene } \\
\text { glycol } \\
\mathrm{cm}^{-1}\end{array}$ & $\begin{array}{l}\text { Diphenyl } \\
\text { acetylene } \\
\mathrm{cm}^{-1}\end{array}$ & $\begin{array}{c}\text { Diphenyl } \\
\text { tetraacetylene } \\
\mathrm{cm}^{-1}\end{array}$ \\
\hline $\begin{array}{ll}28 & 250 \\
27 & 780 \\
27 & 100\end{array}$ & $\begin{array}{l}28300 \\
27830 \\
27150\end{array}$ & $\begin{array}{l}33110 \\
\text { obscured } \\
31750 \\
31500 \\
30770 \\
29370 \\
28410 \\
27030 \\
25710\end{array}$ & 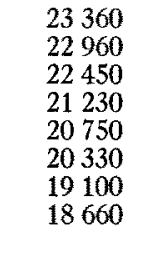 \\
\hline
\end{tabular}

- The polyacetylene glycols used were $\mathrm{CH}_{3}-\mathrm{CHOH}-(\mathrm{C}=\mathrm{C})_{N}-\mathrm{CHOH}-\mathrm{CH}_{s}$.

seems the most honest way of estimating oscillator strengths from working pressures.

Inspection of a number of bands has led to the adoption of $C=0.1$ when $p$ is in mm of mercury and $l$ is in $\mathrm{cm}$.

\section{EXPERIMENTAL}

\section{a. Low Temperature Emission Spectra}

The fluorescence and phosphorescence spectra and the lifetime in the phosphorescent state for several substituted polyacetylenes were determined by well-established methods. ${ }^{3}$

The solvent in all cases was EPA ( 5 parts by volume of isopentane, 5 parts ether, 2 parts ethanol), at $-170^{\circ} \mathrm{C}$. Medium and high pressure mercury lamps were the exciting sources. The filters used were those reported by Kasha. ${ }^{4}$ The phosphoroscope was of the rotating disk type. Lifetime measurements were made photographically with an estimated accuracy of $20 \%$. A Hilger medium quartz spectrograph was used with Kodak IIF and IIN plates developed $3 \frac{1}{4}$ minutes in Ilford ID-2 developer at $68^{\circ} \mathrm{F}$.

The observations are tabulated in Tables II to V. The probable error in the positions of the bands is $\pm 50 \mathrm{~cm}^{-1}$. The phosphorescence spectra are reproduced in Figs. 1

TABLE III. The phosphorescence bands of the aliphatic polyacetylenes.

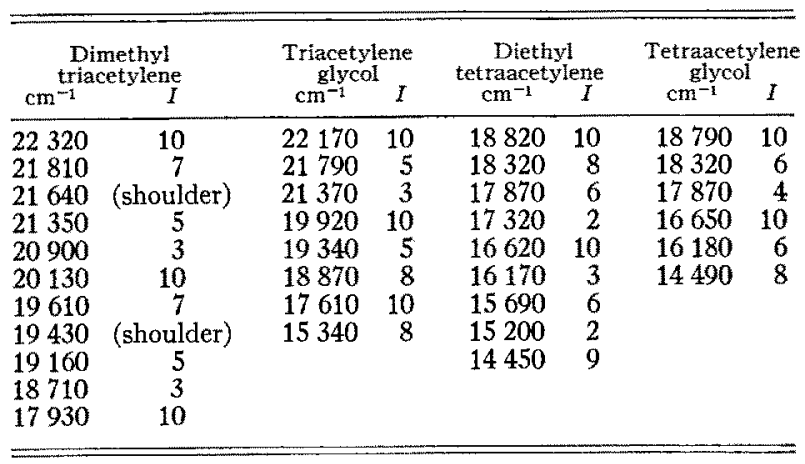

${ }^{3}$ G. N. Lewis and M. Kasha, J. Am. Chem. Soc. 66, 2100 (1944); M. Beer and H. C. Longuet-Higgins, J. Chem. Phys. 23, 1390 (1955).

${ }^{4}$ M. Kasha, J. Opt. Soc. Am. 38, 1068 (1949). and 2. Fluorescence was observed for the four substances listed in Table II. The experimental conditions were such that fluorescence with an intensity of one onehundredth that of the phosphorescence could be detected. No fluorescence was found for diphenyl diacetylene, diphenyl triacetylene, dimethyl triacetylene, and the triacetylene glycol. The oscillator strengths were deduced from the phosphorescence decay lifetimes in the manner described by Lewis and Kasha. ${ }^{5}$

Several attempts were made to detect phosphorescence for dimethyl diacetylene. This compound absorbs appreciably only at wavelengths shorter than $2500 \mathrm{~A}$ and here luminescence work is difficult on account of the relative inefficiency of most light sources, and the increase in scatter loss. Exposures of 30 hours using for source a medium pressure mercury lamp as well as a 5000-volt 0.3 -amp Nichrome spark gave no phosphorescence bands on a fast uv plate.

In a very concentrated solution of dimethyl diacetylene in hexane with $50 \mathrm{~cm}$ path length a very weak band was observed at $28100 \mathrm{~cm}^{-1}$ with $\epsilon_{\max }=0.01$. The upper state located by this absorption band is where, by

TABLE IV. The phosphorescence bands of the diphenyl polyacetylenes.

\begin{tabular}{lrrrrrrrr}
\hline \multicolumn{2}{c}{$\begin{array}{c}\text { Diphenyl } \\
\text { acetylene } \\
\text { cm }^{-1}\end{array}$} & \multicolumn{2}{c}{$\begin{array}{c}\text { Diphenyl } \\
\text { diacetylene } \\
\mathrm{cm}^{-1}\end{array}$} & \multicolumn{2}{c}{$\begin{array}{c}\text { Diphenyl } \\
\text { triacetylene } \\
\text { cm }^{-1}\end{array}$} & \multicolumn{2}{c}{$\begin{array}{c}\text { Diphenyl } \\
\text { tetraacetylene } \\
\text { cm }^{-1}\end{array}$} & $I$ \\
\hline 21870 & 10 & 20270 & 10 & 19380 & 4 & 17150 & 10 \\
20730 & 7 & 19760 & 3 & 18490 & 10 & 16770 & 7 \\
20210 & 7 & 19270 & 4 & 18030 & 5 & 14980 & 9 \\
19610 & 8 & 18660 & 5 & 17610 & 3 & 14660 & 6 \\
19240 & 5 & 18050 & 10 & 17230 & 5 & & \\
18650 & 7 & 17090 & & 16280 & 10 & & \\
18180 & 6 & 16540 & 4 & 15820 & 3 & & \\
17570 & 5 & 15900 & 8 & & & & \\
\hline
\end{tabular}

analogy with the higher members, the phosphorescent state could be expected to lie.

\section{b. The Weak Bands}

In an attempt to locate in the higher members possible analogs of the bands of King and Ingold for acetylene and Woo and Chu for diacetylene, studies were made at high concentration in ethanol, ether, and hexane solutions of dimethyl diacetylene, dimethyl triacetylene, and diethyl tetraacetylene.

The observations are given in Table VI. No new band system could be found for diethyl tetraacetylene.

\section{c. The Gas Phase Spectrum of Dimethyl Triacetylene}

The spectrum of dimethyl triacetylene was measured in the gas phase from 33000 to $80000 \mathrm{~cm}^{-1}$. To 40000 $\mathrm{cm}^{-1}$ a Hilger medium quartz spectrograph was used with a hydrogen discharge as source. The glass absorption cell was $50 \mathrm{~cm}$ long and $5 \mathrm{~cm}$ in diameter with quartz windows. Freshly sublimed dimethyl triacetylene

\footnotetext{
${ }^{6}$ G. N. Lewis and M. Kasha, J. Am. Chem. Soc. 67, 994 (1945).
} 
was sublimed into the tube which was then sealed and suspended in a small oven which made temperature control possible.

At higher frequencies a one meter vacuum spectrograph was used with a 30000 lines per inch grating ruled on glass. Its source was a Lyman discharge tube of the type developed by Collins and Price. ${ }^{6}$

The near ultraviolet spectrum was undoubtedly the vapor phase analog of the bands reported by Jones and co-workers between 2300 and $3100 \mathrm{~A}$.

The far ultraviolet spectrum is reproduced in Fig. 3. The peaks of the bands are given in Table VII. The spectrum is clearly composed of two band systems. The origin of the long wavelength one differs from Jones' intense system by $600 \mathrm{~cm}^{-1}$ and is probably its vapor phase analog.

The shorter wavelength, more intense system, has not been reported before.

TABLE V. The phosphorescence decay lifetimes of the polyacetylenes.

\begin{tabular}{lcc}
\hline Name of compound & $\begin{array}{c}\text { Phosphorescence decay } \\
\text { lifetime in seconds }\end{array}$ & $\begin{array}{c}\text { Oscillator } \\
\text { strength }\end{array}$ \\
\hline Diphenyl acetylene & 0.3 & $2 \times 10^{-8}$ \\
Diphenyl diacetylene & 0.1 & $4 \times 10^{-8}$ \\
Diphenyl triacetylene & 0.04 & $10^{-7}$ \\
Diphenyl tetraacetylene & 0.01 & $6 \times 10^{-7}$ \\
Dimethyl triacetylene & 0.5 & $8 \times 10^{-9}$ \\
Dihydroxy triacetylene & 0.2 & $2 \times 10^{-8}$ \\
Dihydroxy tetraacetylene & 0.03 & $2 \times 10^{-7}$ \\
\hline
\end{tabular}

TABI.E VI. Weak bands of the polyacetylenes.

\begin{tabular}{ccc}
\hline Name of compound & $\mathrm{cm}^{-1}$ & $f$ \\
\hline Dimethyl diacetylene & 36360 & $10^{-5}$ \\
Dimethyl triacetylene & 30340 & $2 \times 10^{-5}$ \\
\hline
\end{tabular}

\section{d. Polarization of Near Ultraviolet Absorption in Single Crystals}

The polarization of the absorption band between $32000 \mathrm{~cm}^{-1}$ and $40000 \mathrm{~cm}^{-1}$ in dimethyl triacetylene was determined using a single crystal. The apparatus and procedure have been described previously. ${ }^{7}$ The crystal was grown from acetone solution under a quartz cover slip on a quartz microscope slide.

It was found that the direction of maximum absorption was the same as the direction of maximum index of refraction. In the crystal the molecules are all parallel to each other ${ }^{1}$ and undoubtedly the index of refraction is greatest along the molecular axis. Thus the absorption is polarized along the axis of the molecule.

\section{DISCUSSION}

Figure 4 summarizes the energy levels of the aliphatic acetylenic compounds. It has been compiled from the data mentioned previously in this paper.

${ }^{6}$ G. B. Collins and W. C. Price, Rev. Sci. Instr. 5, 423 (1934).

${ }_{7}$ W. E. Seeds and M. H. F. Wilkins, Discussions Faraday Soc. 9, 417 (1950).

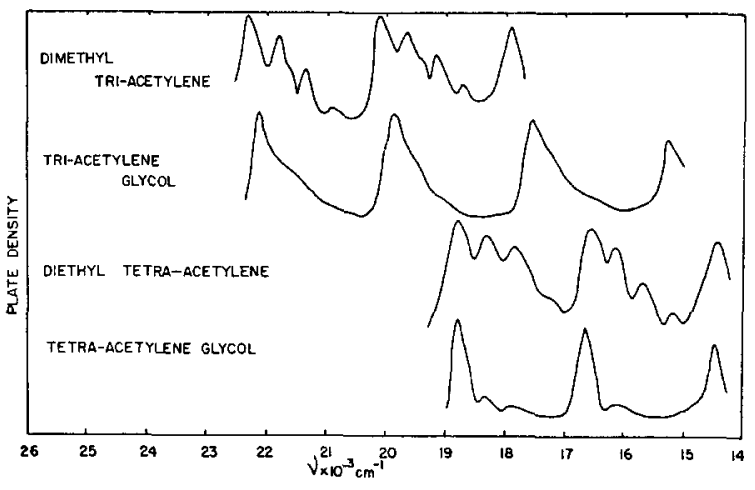

FIG. 1. Phosphorescence bands of the aliphatic polyacetylenes.

The energy levels for $N=1$ are those of acetylene, itself, while for $N=3$ and the higher members only derivatives have been studied. For $N=2$ some of the spectra are those of diacetylene, others of dimethyl diacetylene. Also some of the work has been done in solution and some in the vapor phase; some at room temperature and some at $-170^{\circ} \mathrm{C}$. To make Fig. 4 entirely consistent, corrections would have to be applied. Work on other conjugated compounds suggests that these would be of the order of $1000 \mathrm{~cm}^{-1}$ and would not alter the diagram significantly. No such corrections have been attempted.

The striking similarity of the energy level arrays of the different homologs is inescapable. Corresponding levels are joined with broken lines where data are available. The correlations are supported by the similarity of the intensities of corresponding bands.

The similarity of the vibrational structures of the phosphorescence spectra further confirms that the phosphorescent states correspond. The correspondence with the excited state at $28090 \mathrm{~cm}^{-1}$ in dimethyl diacetylene detected only in absorption, is suggested by its position and intensity.

That the phosphorescent state in organic molecules is a triplet state is now well established. ${ }^{3}$ In the aliphatic polyacetylenes vibrational structure is made up of simple relatively long progressions and the highest frequency band is always very strong. These facts suggest that the

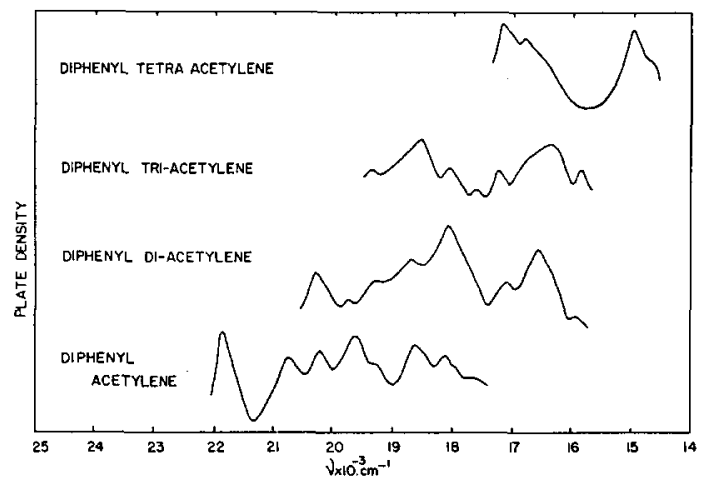

FIG. 2. Phosphorescence bands of the aromatic polyacetylenes. 


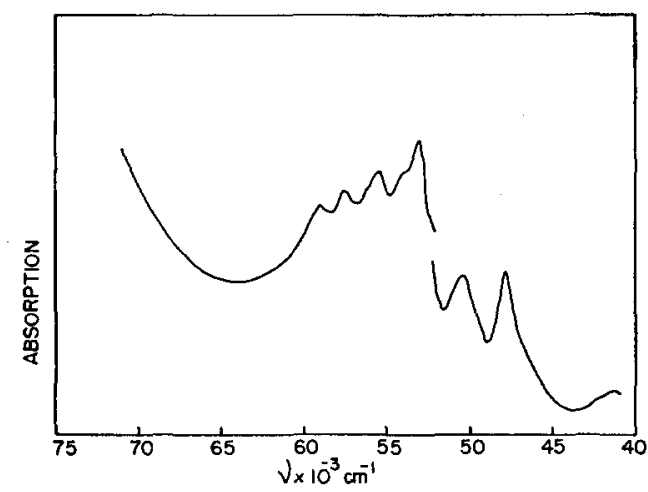

Fig. 3. Vacuum ultraviolet absorption of dimethyl triacetylene.

transition from the phosphorescent state to the ground state is not symmetry forbidden. Then the strongest singlet-triplet absorption would involve this triplet state, and so this is the band most probably detected in absorption. Thus if the diacetylene level is a triplet as is strongly suggested by its intensity, it corresponds to the phosphorescent levels.

It is seen from Fig. 4 that the separation of the $S_{1}$ and $S_{2}$ levels decreases as $N$ increases. For $N=4$ no $S_{1}$ level was observed, suggesting that it is obscured by the $S_{2}$ level. For $N=5 S_{1}$ is probably higher than $S_{2}$.

It is well known that, if the probability of absorption of radiation of a particular frequency is high, then so is the probability of its reverse, the process of emission from the excited state. ${ }^{5}$ Therefore, it is clear from the intensities of the absorption bands that the probability of radiative transition from the $S_{2}$ states is much greater than from the $S_{1}$ states. Thus, since the fluorescent state is usually the lowest singlet state, one would expect those acetylenic molecules to fluoresce readily, in which the $S_{2}$ and not the $S_{1}$ state is the lowest. This is exactly what was found: triacetylene showed only phosphorescence, while tetraacetylene also fluoresced.

Even with the diphenyl polyacetylenes fluorescence was observed for $N=4$ and not for $N=2$ and $N=3$. Thus these substances too behaved as the aliphatic polyacetylenes. Diphenyl monoacetylene, however, fluoresced; but it is perhaps not surprising that here the two aromatic groups dominate the acetylenic character.

The correspondence of the $32700 \mathrm{~cm}^{-1}$ band system in dimethyl triacetylene with the $40000 \mathrm{~cm}^{-1}$ band of dimethyl diacetylene has already been proposed. ${ }^{2}$ This

TABLE VII. Bands of dimethyl triacetylene.

\begin{tabular}{ll}
\hline$\tilde{\nu} \mathrm{cm}^{-1}$ & \multicolumn{1}{c}{1} \\
\hline 47750 & Strong \\
50302 & Strong \\
53160 & Very strong \\
53650 & Very strong \\
55520 & Very strong \\
56090 & Very strong \\
58837 & Very strong \\
\hline
\end{tabular}

is amply confirmed by similarity of intensity and vibrational structure. In both these substances there are two absorption regions, to shorter wavelengths. In each case the more intense one is the short wavelength region. Thus the correspondence of these two regions is indicated as shown in Fig. 4. It follows that Price's Rydberg bands $(S 4)$ do not correlate with Jones' intense bands (S3).

Extrapolation of the wavelength of the $S_{4}$ bands to hexa- and hepta-acetylene might lead one to expect their appearance in the quartz region. However, no absorption system in addition to Jones' bands has been detected. This is taken to mean that the Rydberg bands are not observable in solution.

The presence of two band systems, $S_{2}$ and $S_{3}$, in acetylene was reported by Rose ${ }^{8}$ but not by Herzberg ${ }^{9}$

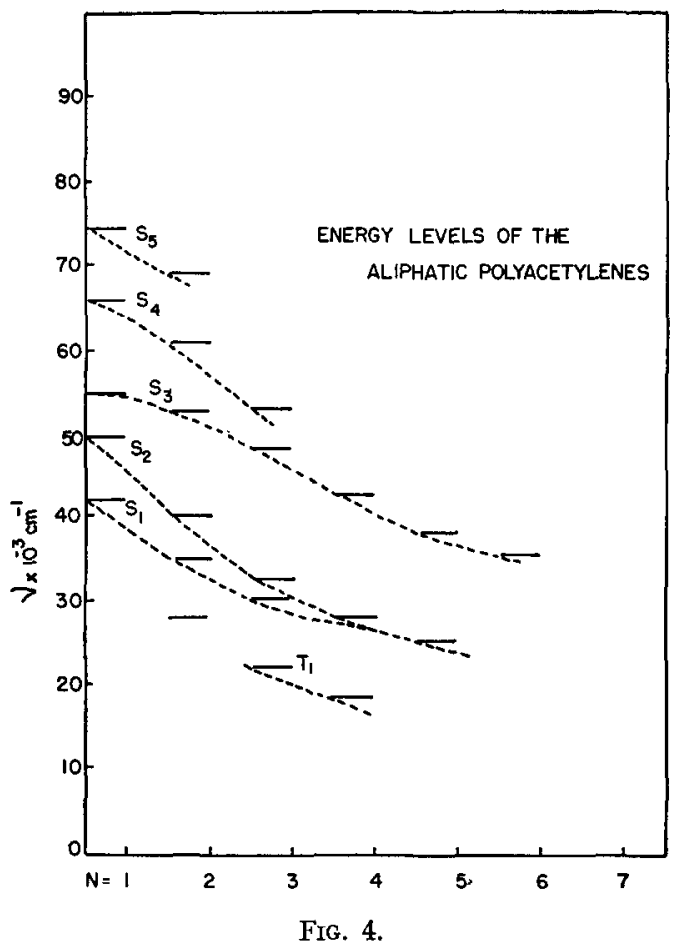

or Price ${ }^{10}$ who have also studied that region of the spectrum. If Rose's claim is incorrect then the $S_{3}$ level of acetylene is probably at higher energy but obscured by the perpendicular Rydberg bands $S_{4}$. This latter view is suggested by recent calculations of Longuet-Higgins and McEwen. ${ }^{11}$

\section{ASSIGNMENT OF EXCITED STATES OF ALIPHATIC POLYACETYLENES}

It will now be shown that on the basis of molecular orbital theory a similarity between the spectra of the polyacetylenes is to be expected, and an attempt will be

${ }^{8}$ A. Rose, Z. Physik 81, 751 (1933).

${ }^{9}$ G. Herzberg, Trans. Faraday Soc. 27, 378 (1931).

${ }^{10}$ W. C. Price, Phys. Rev. 47, 444 (1935).

${ }^{11}$ H. C. Longuet-Higgins and L. McEwen (to be published). 
made to identify the observed energy levels with theoretically predicted ones of particular symmetry.

As a first approximation it will be assumed that the molecules are linear. Though this is not always so in the excited states, ${ }^{12}$ the arguments which follow would be only slightly changed by taking bending into consideration.

The $\pi$-electron systems of the polyacetylenes may be represented as in Fig. 5. If $N$ is the number of triple bonds, then there are $4 N \pi$ electrons distributed through $2 N$ doubly degenerate $\pi$ orbitals of which $N$ are bonding and $N$ antibonding. In every molecule the symmetries of the orbitals are alternately $\pi_{u}, \pi_{\sigma}$, the lowest being always $\pi_{u}$. In the ground state of the molecules all the bonding orbitals are just filled and the molecular symmetry is ${ }^{1} \Sigma_{g}{ }^{+}$. If $N$ is even, the highest filled orbital is a $\pi_{o}$ and the lowest empty one a $\pi_{u}$. If $N$ is odd the highest filled orbital is a $\pi_{u}$ and the lowest empty one a $\pi_{\theta}$. In both cases the promotion of an electron from the highest occupied to the lowest unoccupied level leads to one of three states: $\Sigma_{u}-, \Sigma_{u}{ }^{+}, \Delta_{u}$.

The possible transitions for the acetylenes will now be discussed.

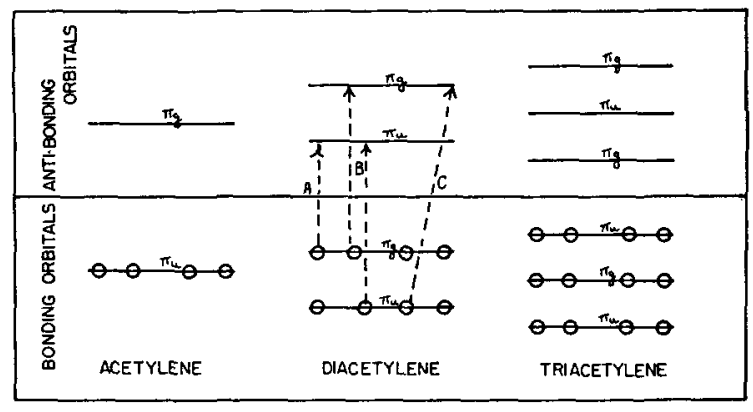

Fig. 5. The $\pi$-electron systems of the first three aliphatic polyacetylenes.

\section{a. Transitions Involving Jumps from the Highest Occupied to the Lowest Unoccupied Orbital}

(1) ${ }^{1} \Sigma_{g}+\rightarrow^{3} \Sigma_{u}{ }^{+}$.

The transition is spin forbidden and Platt ${ }^{13}$ associates with this a "forbiddenness factor" of $10^{-5}$. Thus the oscillator strength would be of the order of $10^{-5}$ to $10^{-6}$ and the radiative decay lifetime of the excited state would be of the order of $10^{-2}$ seconds. Ross ${ }^{14}$ has made calculations of the energy levels of acetylene and using a number of different approximations always predicted least energy for the ${ }^{3} \Sigma_{u}{ }^{+}$state. This state would then be the phosphorescent state. Although the phosphorescent state in acetylene has not yet been observed, it is believed that ${ }^{3} \Sigma_{u}+$ is the symmetry of the $T_{1}$ states in the higher homologs.

${ }^{12}$ C. K. Ingold and G. W. King, Nature 169, 1101 (1952); J. Chem. Soc. 1953, 2702-2755.

${ }_{13}$ J. R. Platt, J. Opt. Soc. Am. 43, 252 (1953).

14 I. G. Ross, Trans. Faraday Soc. 48, 973 (1952).
The vibrational progressions in the phosphorescence spectra of the aliphatic polyacetylenes comprise frequencies of about $2200 \mathrm{~cm}^{-1}$ and $480 \mathrm{~cm}^{-1}$. Such frequencies in diacetylene have been assigned by Vallance Jones ${ }^{15}$ to a $\Sigma_{g}{ }^{+}$species triple bond stretching mode and a $\Pi_{o}$ species bending frequency respectively. Assuming a ${ }^{1} \Sigma_{g}+$ ground state, the progressions can be built up of $\Sigma_{g}{ }^{+}$and $\Pi_{v}$ states alone. If the phosphorescence is not a symmetry forbidden transition then the upper state must be $\Sigma_{u}{ }^{+}$or $\Pi_{u}$. Thus the vibrational structure is consistent with this assignment. A spin forbidden transition would almost certainly have a more complicated vibrational structure.

$$
\text { (2) } 1 \Sigma_{v}+\rightarrow^{3} \Delta_{u}
$$

The transitions are spin forbidden and momentum forbidden. Thus they would probably be about $10^{2}$ times weaker than the above. Ross's calculations suggest that the excited states are not the lowest triplets. There would be little chance of observing them in absorption spectra and none at all in emission spectra since radiationless transitions would lead to degradation to the lower triplet long before the improbable emission occurred.

$$
\text { (3) } 1 \Sigma_{g}{ }^{+} \rightarrow^{3} \Sigma_{u}{ }^{-} \text {. }
$$

The transitions are spin forbidden as well as +- forbidden. Ross's calculations suggest that these excited states too lie above the ${ }^{3} \Sigma_{u}$ states. Their observation is as unlikely as the above.

$$
\text { (4) } 1 \Sigma_{g}^{+} \rightarrow 1 \Sigma_{u}^{-} \text {. }
$$

The transitions are +- forbidden and will not be strong. By all of Ross's calculations the excited states are the lowest singlets. If the excited molecule becomes bent into one of symmetry $C_{2 h}$ the state merges into one of symmetry $A_{u}$. The transition ${ }^{1} \Sigma_{g}{ }^{+} \rightarrow A_{u}$ is allowed but only to the extent to which the bending of the molecule changes the electronic wave function. This band is certainly the one studied for acetylene by King and Ingold and the corresponding levels in the higher members of the series are the $S_{1}$ levels.

$$
\text { (5) }{ }^{1} \Sigma_{g}{ }^{+} \rightarrow 1 \Delta_{u} \text {. }
$$

The transitions are momentum forbidden. Platt associates with this a forbiddenness factor $10^{-1}$ to $10^{-3}$. The oscillator strength will then be of the order of $10^{-2}$. By all of Ross's calculations the excited states lie near but slightly above the ${ }^{1} \Sigma_{u}-$ level. Both intensity and position suggest the $S_{2}$ levels of Fig. 4 .

The interpretation of the parallel polarization of the band is the following. The forbidden transition to the ${ }^{1} \Delta_{u}$ state cannot take place. However transitions to a somewhat distorted ${ }^{1} \Delta_{u}$ state may be allowed. If a linear molecule whose electronic wave function is of ${ }^{1} \Delta_{u}$ species becomes bent so that the nuclei have symmetry $C_{2 h}$ then the state of the molecule becomes either ${ }^{1} A_{u}$ or

\footnotetext{
${ }^{15}$ A. V. Jones, Proc. Roy. Soc. (London) A211, 285 (1952).
} 
${ }^{1} B_{u}$. If it is bent so that the nuclei have symmetry $C_{2 v}$ then the molecule becomes either ${ }^{1} A_{2}$ or ${ }^{1} B_{1}$.

Transitions from the $1 \Sigma_{g}+$ ground state are allowed to the ${ }^{1} A_{u}$ state with electric vector perpendicular to the plane of the molecule, allowed to the ${ }^{1} B_{u}$ state with electric vector in the plane of the molecule, forbidden to the state ${ }^{1} A_{2}$, and allowed to the state ${ }^{1} B_{1}$ with electric vector parallel to the axis of the molecule.

Thus the parallel polarization of the band implies that the excited state is either ${ }^{1} B_{u}$ or ${ }^{1} B_{1}$.

The excited molecule may either be naturally bent in the excited state or only during a vibration.

$$
\text { (6) }{ }^{1} \Sigma_{g}{ }^{+} \rightarrow{ }^{3} \Delta_{g} \text {. }
$$

This transition is spin forbidden, momentum forbidden and parity forbidden.

$$
\text { (7) }{ }^{1} \Sigma_{g}{ }^{+} \rightarrow{ }^{3} \Sigma_{g}-
$$

This transition is spin forbidden, even-odd forbidden and +- forbidden.

$$
\text { (8) }{ }^{1} \Sigma_{g}+\rightarrow^{1} \Gamma_{g} \text {. }
$$

This is a two electron transition highly momentum forbidden and even-odd forbidden.

$$
\text { (9) } 1 \Sigma_{g}+{ }^{+} \Delta_{g} \text {. }
$$

This is a two electron transition which is momentum and even-odd forbidden. The last four transitions will hardly be detected for they are weaker than others which overlap them and will not emit radiation since they are not the lowest levels of that multiplicity.

$$
\text { (10) } 1 \Sigma_{g}{ }^{+} \rightarrow 1 \Sigma_{u}{ }^{+} \text {. }
$$

These are the only fully allowed one electron subRydberg transitions. In the higher members the intense ${ }^{12}$ bands measured by Jones et al. are undoubtedly the longest wavelength allowed transitions. The upper states are the $S_{3}$ states of Fig. 4 .

\section{b. Transitions Involving Jumps from the Second Highest Occupied to the Lowest Unoccupied Orbital and Transitions from the Highest Oc- cupied to the Second Unoccupied Orbital}

These transitions involve either a jump from one $\pi_{g}$ orbital to another or from one $\pi_{u}$ orbital to the other. In both cases the upper states of the molecule must be $\Sigma_{g}^{+}, \Sigma_{g}^{-}$or $\Delta_{u}$. The transitions to these states are weaker and at shorter wavelengths than the transitions to the corresponding " $u$ " states; and the bands will be obscured by the much stronger bands.

\section{c. Transitions Involving Electron Jumps from the Second Highest Occupied to the Second Unoccupied Orbital}

These transitions involve a jump from $\pi_{u}$ to a $\pi_{b}$ or from a $\pi_{g}$ to a $\pi_{u}$ orbital and the expected selection rules will be similar to those described in Type $a$ transitions. The excited states however will lie very high above the ground states and the bands will not be found in the long wavelength portion of the spectrum.

It follows from the above that the most probable interpretation of the energy level diagram is that

$$
\begin{aligned}
& T_{1} \text { states are }{ }^{3} \Sigma_{u}{ }^{+} \\
& S_{1} \text { states are }{ }^{1} \Sigma_{u}{ }^{-} \\
& S_{2} \text { states are }{ }^{1} \Delta_{u} \\
& S_{3} \text { states are }{ }^{1} \Sigma_{u}{ }^{+} .
\end{aligned}
$$

$S_{4}$ and $S_{5}$ are perpendicular and parallel Rydberg bands. The symmetries of the excited states have already been discussed by Mulliken. ${ }^{16}$

\section{REMARKS ON THE AROMATIC POLYACETYLENES}

The energy level diagram for the diphenyl polyacetylenes is given in Fig. 6 . It is based on results of Schlubach and Frazen. ${ }^{17}$

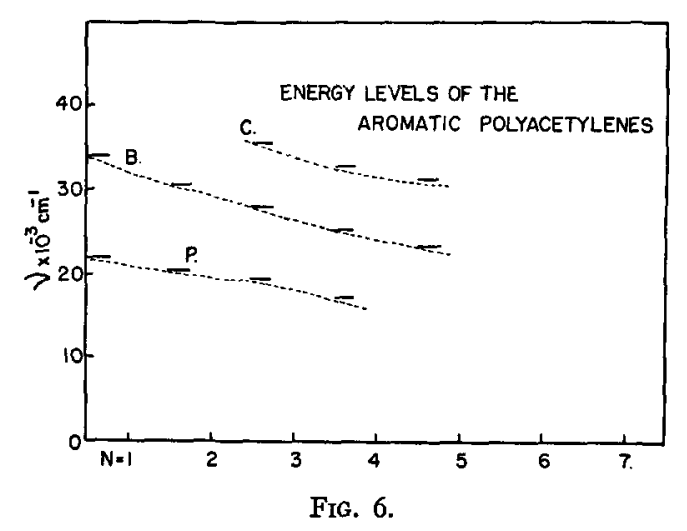

The available information for these aromatic polyacetylenes is much less than for the aliphatic ones and so the conclusions must be less definite. A similarity of the $B$ and $C$ energy levels of Fig. 6 with the $S_{2}$ and $S_{3}$ levels of Fig. 4 should be noted however. The intensities and relative positions are comparable, though the energy levels of the aromatic members are lower. Thus, the spectra are those of perturbed polyacetylenes. This view is further supported by the occurrence in all the emission spectra of a $2200 \mathrm{~cm}^{-1}$ vibrational structure characteristic of the carbon carbon triple bond stretching.

\section{ACKNOWLEDGMENTS}

The author would like to thank Professor H. C. Longuet-Higgins for attracting his attention to the polyacetylenes and for many helpful discussions, Professor W. C. Price for his hospitality and help during the measurement of the far ultraviolet spectra, and Dr. W. E. Seeds for cooperation in the work with polarized light.

${ }^{16}$ R. S. Mulliken, J. Chem. Phys. 3, 517 (1935).

${ }_{17}$ H. H. Schlubach and V. Frazen, Ann. Chem. 573, 110 (1951). 